\title{
Bilateral vocal cord paralysis following coronary artery bypass surgery
}

\author{
Seong Mi Yang ${ }^{1}$, Jin-Young Hwang ${ }^{2}$, Jee-Eun Chang ${ }^{2}$, and Kyoung-Beom Min ${ }^{2}$ \\ Department of Anesthesiology and Pain Medicine, ${ }^{1}$ Seoul National University Hospital, ${ }^{2}$ SMG-SNU Boramae Medical Center, Seoul, \\ Korea
}

We report a case of bilateral vocal cord paralysis (VCP) that occurred after coronary artery bypass surgery and we review the mechanisms of VCP and the preventable factors. We emphasize to consider VCP if respiratory insufficiency occurs following extubation in cardiac surgery, and an early diagnosis is sure to prevent life-threatening problems such as airway compromise and aspiration pneumonia.

A 66-year-old female patient $(153.4 \mathrm{~cm}, 46.8 \mathrm{~kg})$ was scheduled for a coronary artery bypass surgery. She had a medical history of hypertension, coronary artery disease, and diabetes mellitus. Recent coronary angiography showed progressed lesions at the left anterior descending and left circumflex arteries. After the patient was transferred to the operating room and an arterial cannulation was done, anesthesia was induced. Tracheal intubation was performed with a $7.0 \mathrm{~mm}$-internal diameter endotracheal tube (SoftVent ${ }^{\circledR}$ Pro, HVLP-Cuff, Murphy, Unomedical, Malaysia) using a direct curved laryngoscope. The balloon was inflated until the cuff pressure was measured as $25 \mathrm{cmH}_{2} \mathrm{O}$ by a handheld aneroid manometer (VBM, Germany). The cuff was palpated at the suprasternal notch and the endotracheal tube was fixed at $20 \mathrm{~cm}$ from the incisors. An ultrasound guided insertion of a central venous cannula was performed in the right internal jugular vein and a transesophageal echocardiographic (TEE) probe (diameter: $10.5 \mathrm{~mm}$, width of tip: $14.5 \mathrm{~mm}$; TEV5Ms, Siemens Medical Solutions, Mountain View, USA) was inserted without difficulty. After median sternotomy, the left internal mammary artery was harvested and off-pump coronary artery bypass surgery was performed. The cuff pressure of the endotracheal tube was intermittently measured maintaining the intracuff pressure at $25 \mathrm{cmH}_{2} \mathrm{O}$ during the operation and the post operation period, and the fraction of inspired oxygen was maintained below 0.5 using medical air and oxygen.

A planned extubation was performed after ventilator care for 2 days. Immediately after extubation, the patient complained of respiratory difficulties and an inspiratory stridor was detected. Dexamethasone $5 \mathrm{mg}$ was given and respiration was assisted with a bag-valve-mask for several minutes. The respiratory difficulties were relieved and the stridor disappeared. Chest Xray findings and arterial blood gas analysis were within normal range. Methylprednisolone $25 \mathrm{mg} /$ day was administered due to the suspicion of laryngeal edema and spontaneous breathing was maintained without events under oxygen $10 \mathrm{~L} / \mathrm{min}$ via a facial mask. One day after extubation the patient complained of hoarseness. The otorhinolaryngeal department was consulted for a vocal cord examination and a gap was shown between the vocal cords and both vocal folds were shown to be immobile. Feeding through a nasogastric tube was started and the patient was kept under close observation. The patient had no respiratory difficulties even though a minimal stridor was detected intermittently. On the 18th post-operative day, the videofluoroscopic swallowing study showed an aspiration at the pharyngeal stage and the patient was put on rehabilitation for dysphagia. On the 20th post-operative day, the fiberoptic endoscopic evaluation of swallowing (FESS) showed improved movement of both vocal cords and no aspiration signs. The patient was discharged one week later and after three months, her voice was normalized and

Corresponding author: Jee-Eun Chang, M.D., Department of Anesthesiology and Pain Medicine, SMG-SNU Boramae Medical Center, 20 Boramae-ro 5-gil, Dongjak-gu, Seoul 156-707, Korea. Tel: 82-2-870-2864, Fax: 82-2-747-5639, E-mail: sw411528@hanmail.net

(c) This is an open-access article distributed under the terms of the Creative Commons Attribution Non-Commercial License (http:// creativecommons.org/licenses/by-nc/3.0/), which permits unrestricted non-commercial use, distribution, and reproduction in any medium, provided the original work is properly cited. 
the FESS showed no abnormal findings.

VCP results from a direct vocal cord injury or a recurrent laryngeal nerve (RLN) paralysis following cardiac surgery. Direct manipulation and retraction of the heart, median sternotomy, as well as an excessive sternal traction for harvesting of the internal mammary artery may be a cause of RLN paralysis related to the anatomy of RLN [1]. The right RLN crosses the subclavian artery, winds the artery backward, and ascends in the tracheoesophageal groove. The left RLN crosses the aortic arch and reaches the tracheoesophageal groove [1]. Hypothermic injury with ice/slush collecting in the pleural cavity in close proximity to the left RLN has been also reported to contribute to RLN paralysis [2]. The repeated and unsuccessful attempts of inserting TEE probe can compress the nerve at its entry into the larynx, resulting in RLN paralysis [3]. During central venous catheterization, direct RLN injury is possible because the catheterized vessels are close to the RLN. In addition, traumatic endotracheal intubation can cause a direct mechanical injury such as a cricoarytenoid dislocation, and an excessively inflated endotracheal tube cuff can compress the RLN or its anterior branch at the tracheoesophageal groove [4]. In this case, the chest $\mathrm{x}$-ray and manometer findings checked during the intubation period show that endotracheal tube location and cuff pressure were within a normal range. The central line, TEE, and the endotracheal tube also were all inserted without any difficulty and events. Therefore, controllable or intubation related factors can be excluded. We suppose that the causes of VCP in this case were the factors associated with the heart operation such as excessive sternal traction and direct manipulation of the heart.

The clinical manifestations of a bilateral VCP are respiratory insufficiency, stridor, and hoarseness. In this case, the patient had respiratory difficulties and stridor immediately after extubation and the symptoms subsided after steroid injection. The day after extubation, the patient complained of hoarseness and was diagnosed with bilateral VCP. This shows that laryngeal spasm or edema that causes respiratory difficulties and stridor immediately after extubation [5] could be accompanied by bilateral VCP in this case. Generally, bilateral VCP is clinically fatal and prompt management such as re-intubation and mechanical ventilation can be required because the vocal cords can be immobile while being closed. In our case, a gap existed between the vocal folds. Therefore adequate ventilation and oxygenation was possible via the gap. However, re-intubation and mechanical ventilation under the suspicion of bilateral VCP should have taken place if the stridor or respiratory symptoms had still existed after the use of steroid or the assistance of ventilation.

It is not easy to prevent a VCP in cardiac surgery, because there are several contributing factors for the occurrence. We should take effort to avoid any controllable risk factors, including abnormal tube size and location, high intra-cuff pressure, and traumatic insertion of TEE probe or central venous catheter. Also, a VCP must be suspected if patients have hoarseness, stridor, and respiratory difficulties after extubation.

\section{References}

1. Hodgman JR, Cosgrove DM. Post-hospital course and complications following coronary artery bypass surgery. Cleve Clin Q 1976; 43: 125-9.

2. Titche LL. Causes of recurrent laryngeal nerve paralysis. Arch Otolaryngol 1976; 102: 259-61.

3. Zwetsch G, Filipovic M, Skarvan K, Todorov A, Seeberger MD. Transient recurrent laryngeal nerve palsy after failed placement of a transesophageal echocardiographic probe in an anesthetized patient. Anesth Analg 2001; 92: 1422-3.

4. Wason R, Gupta P, Gogia AR. Bilateral adductor vocal cord paresis following endotracheal intubation for general anaesthesia. Anaesth Intensive Care 2004; 32: 417-8.

5. Wittekamp BH, van Mook WN, Tjan DH, Zwaveling JH, Bergamans DC. Clinical review: post-extubation laryngeal edema and extubation failure in critically ill adult patients. Crit Care 2009; 13: 233. 